\title{
Tofacitinib Blocks Entheseal Lymphocyte Activation and Mod- ulates MSC Adipogenesis but Does not Directly Affect Chondro- and Osteogenesis
}

\author{
Tobias Russell 1,*, Hannah Rowe 1,*, Charlie Bridgewood 1, Richard J. Cuthbert ${ }^{1}$, Abdulla Watad 1,3, Darren New- \\ ton ${ }^{2}$, Elena Jones ${ }^{1}$, Dennis McGonagle ${ }^{1}$ \\ 1 Leeds Institute of Rheumatic and Musculoskeletal Medicine (LIRMM), University of Leeds, Leeds, UK \\ 2 Division of Hematology and Immunology, Leeds Institute of Medical Research at St. James's, University of \\ Leeds, Leeds, UK \\ 3 Department of Medicine B and Zabludowicz Center for Autoimmune Diseases, Sheba Medical Center, Tel- \\ Hashomer, Ramat-Gan, Israel, Sackler Faculty of Medicine, Tel-Aviv University, Tel-Aviv, Israel \\ * Correspondence: meddgm@leeds.ac.uk
}

\begin{abstract}
Entheseal spinal inflammation and new bone formation with progressive ankylosis may occur in ankylosing spondylitis (AS) and psoriatic arthritis (PsA). This study evaluated whether JAK inhibition with tofacitinib modulated the key disease associated cytokines, TNF and IL-17A and whether tofacitinib also modulated bone marrow stromal cell-derived mesenchymal stem cells (MSCs) function including osteogenesis, since post inflammation new bone formation occurs in these conditions. Methods: Conventional entheseal derived $\alpha \beta \mathrm{CD} 4+$ and CD8+ T-cells were investigated following anti-CD3/CD28 bead stimulation to determine IL-17A and TNF levels in Tofacitinib treated $(1000 \mathrm{nM})$ peri-entheseal bone (PEB) and peripheral blood mononuclear cells (PBMC) following ELISA. Bone marrow stromal cell-derived mesenchymal stem cells (MSCs) colony forming unit (CFU-F) and multilineage potential was evaluated using tofacitinib (dosages ranging between 100, 500, 1000 and 10000nM). Results: Induced IL-17A and TNF cytokine production from both entheseal CD4+ T-cells and CD8+ T-cells were effectively inhibited by tofacitinib. Tofacitinib treatment did not impact on CFU-F potential or in vitro chondro- and osteogenesis. However, tofacitinib stimulation increased MSC adipogenic potential with greater Oil Red O stained area. Conclusion: Inducible IL-17A and TNF production by healthy human entheseal CD4+ and CD8+ T-cells was robustly inhibited in vitro by tofacitinib. However, tofacitinib did not impact on MSC osteogenesis but stimulated in vitro MSC adipogenesis, the relevance of which needs further evaluation given the adipocytes are associated with new bone formation in SpA.
\end{abstract}

Keywords: tofacitinib; JAK-STAT; chondrogenesis

\section{Introduction}

The seronegative spondyloarthropathies (SpA) encompass AS, its non-radiographic predecessor and PsA including peripheral and axial disease. Cytokines including TNF and IL-17A are pivotal in the pathogenesis of the seronegative SpA in both experimental settings and more importantly in humans, as demonstrated by the success of cytokine targeting in humans [1]. A peculiar aspect of AS therapy, especially under anti-TNF agents was progressive new bone formation in the initial phases. Short-term control of inflammation appears insufficient to stop the progress of ankyloses especially in animal models [2].

Skeletal tissue repair and remodeling responses are orchestrated by tissue-resident stromal cells including mesenchymal stem cells (MSCs) which have been identified in the synovium, periosteum, bone marrow and synovial fluid [3-7] and have been reported at the entheses which are the target sites of new bone formation $[8,9]$. MSC's have been 
closely studied due to their ability to differentiate into numerous lineages including osteocyte, chondrocyte, and adipocyte [10]. In recent years it has been established that animal models of SpA have resident immune cell populations at the enthesis and in particular immune cells that produce abundant IL-17A and IL-22 [11,12], where both cytokines can modulate aberrant bone formation reactions. The human enthesis also contains both innate and adaptive immune cell populations that are capable of inducible TNF and IL-17A production, such as Gamma Delta, CD4 and CD8+ T-cells [12,13]. Animal models of SpA, as well as showing new bone formation also show decreased BMAT (Bone marrow adipose tissue)[14].

The JAK kinase inhibitors have emerged as important targets in the SpA group of diseases[15] and it is interesting that neither TNF nor IL-17A signal directly through the JAK pathways. Tofacitinib is a highly selective Janus kinase (JAK)- 1 and JAK3 and also to a lesser extent, tyrosine kinase 2 (TYK2) inhibitor, licensed for rheumatoid arthritis (RA) [16-18] and PsA [19]. JAKs are integral to a number of cytokine and growth factor signaling cascades [20] that may affect TNF and IL-17A production indirectly. The use of JAKi is expanding in the SpA arena and efficacy is as good as the anti-TNF agents [21]. It has previously been show that Tofacitinib inhibits T-cell derived IL-17A, but not previously at the enthesis[22].

The impact of JAK inhibition with MSC differentiation could impact on tissue repair and new bone formation. For example, tofacitinib treatment inhibited bone-marrow derived fibroblasts into myofibroblasts in vitro differentiation [23]. A recent study showed that tofacitinib augmented human MSC osteogenesis that was noted to be relevant for bone erosion in RA [24]. In the context of joint disease, the treatment of osteoarthritic MSCs with tofacitinib during chondrogenic differentiation in vitro suggested that it could, in part, rescue the reduction of glycosaminoglycan content [25]. Hence, JAK inhibition could theoretically affect stromal tissue responses in the joint organ in different chronic joint diseases in addition to its impact on immune cells.

In this study, we evaluated the in vitro effects of tofacitinib on tissue responses in the enthesis including its impact on entheseal lymphocyte activation and MSC function. In an in vitro enthesitis model, we showed that it robustly suppressed conventional $\alpha \beta$ CD4+ and CD8+ T-cell activation and attenuated entheseal derived IL-17A and TNF production. Our data suggests that tofacitinib had no direct effects on in vitro marrow MSC osteogenesis but stimulated adipogenesis, the latter observation being of interest given the role of fat in axial new bone formation in SpA [26].

\section{Materials and Methods}

\subsection{Patient Consent and collection of samples}

Written informed consent was given by all patients and research was carried out in compliance with the Helsinki Declaration. Ethics committee approval was obtained from the Leeds East Ethics Committee under permit number 06/Q1206/127. Bone marrow (BM) aspirates were taken from the Iliac crest of patients with acute trauma patients or undergoing elective orthopaedic surgery [27]. Entheseal samples collection was approved by the North West - Greater Manchester West Research Ethics Committee (REC: 16/NW/0797) on 16 November 2016.

\subsection{Isolation of Primary Cells from Enthesis and Matched Blood}

Entheseal samples were separated into entheseal soft tissue (EST) and peri-entheseal bone (PEB) and both were enzymatically digested as previously described [11]. For both cell preparations, blood and entheseal cells, density gradient separation (Lymphoprep) was conducted to obtain peripheral blood mononuclear cells (PBMCs) and entheseal mononuclear cells (EMCs), respectively, using methods previously described [28].

\subsection{Magnetic Cell Separation}


Following isolation of EMCs from digested entheseal samples and also PBMCs from processed blood, CD4+ and CD8+ T-cells were subsequently isolated using biotinylated anti-CD4 or CD8 antibodies (both from Miltenyi Biotech). Cells were isolated using magnetic separation (Miltenyi Biotech LS columns), according to the manufacturer's instructions.

\subsection{Cell Preparation}

Following cell isolation, CD4+ and CD8+ T-cells were plated out in a 96-well-plate (minimum of $5 \times 10^{4}$ cells/well) in RPMI (GIBCO) containing 10\% fetal calf serum (FCS) and $1 \%$ penicillin/streptomycin.

For inhibition studies, cells were incubated Tofacitinib 1000nM (Pfizer) and DMSO control $(0.1 \%)$. Tofacitinib was diluted to maintain a final concentration of $0.1 \%$ DMSO. Cells were stimulated using anti-CD3/CD28 (GIBCO) for $48 \mathrm{hr}$.

\subsection{TNF and IL-17A Determination by ELISA in Entheseal Stimulated Cell Supernatants}

Following $48 \mathrm{hr}$ stimulation, cells were removed by centrifugation and supernatant was frozen and stored at $-80^{\circ} \mathrm{C}$. Concentrations of TNF and IL-17A were quantified using sandwich ELISAs from eBioscience/ThermoFisher. ELISAs were carried out according to the manufacturer's protocol. Following this, $\mathrm{pg} / \mathrm{mL}$ and $\mathrm{pg} / \mathrm{cell}$ were calculated.

\subsection{CFU-F Assay and Measurement of Colony Area}

The CFU-F assay performed was a modification of the method described by Galotto et al [29]. BM aspirate $(100 \mu \mathrm{l})$ was seeded, in duplicate, in $100 \mathrm{~mm}$ diameter tissue culture dishes in StemMACS media (Miltenyi Biotec). Tofacitinib was added in concentrations of 100nM, 500nM, 1000nM, and 10,000nM. Alternatively, Dimethyl sulfoxide (DMSO) was used as carrier control, an unstimulated control was also included. Assessment of colony number and measurement of colony size then proceeded as previously described [27].

\subsection{In Vitro Osteogenesis, Chondrogenesis and Adipogenesis}

Following the removal of erythrocytes, BM aspirates were seeded at a density of $8 \times 10^{4}$ cells $/ \mathrm{cm}^{2}$ into tissue culture grade flasks (Corning) with StemMACS media and incubated in standard culture conditions $\left(5 \% \mathrm{CO}_{2}, 37^{\circ} \mathrm{C}\right)$. Adherent cells were expanded to $<80 \%$ confluence then passaged, re-seeded at $4.5 \times 10^{3}$ cells $/ \mathrm{cm}^{2}$ and returned to culture, up to 3 passages were performed. Osteogenic differentiation and assessment of alkaline phosphatase activity, as well as matrix mineralization by measurement of calcium accumulation, was measured as previously described [30,31] at 14 and 21 days after initiation of osteogenic conditions respectively. Osteogenic differentiation media was supplemented with 500, 1000 or 10,000nM tofacitinib or carrier control, DMSO. For assessment of chondrogenesis cell pellets were incubated in chondrogenic conditions for 21 days and glycosaminoglycan (GAG) accumulation was visualized histologically as well as measured quantitatively as previously described [30,31]. Adipogenic differentiation was performed over 14 days and visualized by oil red staining as previously described [30,31], photomicrographs were captured using a CKX41 inverted light microscope and analyzed using image analysis software NIS elements (Nikon). Adipogenic differentiation and quantitative measurement was performed over 14 days using nile red fluorescence and measured using a Mithras LB940 plate reader (Berthold) as previously described [32]. In all cases, differentiation media was supplemented with $500 \mathrm{nM}, 1000 \mathrm{nM}$, or 10,000nM tofacitinib or DMSO carrier control.

To further explore the effect of tofacitinib on adipogenesis, adipogenic differentiation was repeated over a period of 21 days and concentrations of $10 \mathrm{nM}, 50 \mathrm{nM}, 100 \mathrm{nM}$, and $500 \mathrm{nM}$, as well as a DMSO control, were added to the differentiation media.

\subsection{Statistics}


For the in vitro data sets, the Shapiro-Wilk normality test was used to assess distribution normality and to determine appropriate correlation and significance testing. For data sets which contained fewer than 6 data points per group, a non-gaussian distribution was assumed. Statistical significance is defined as $\mathrm{p}<0.05:{ }^{*}$ indicates $\mathrm{p}<0.05,{ }^{* *}$ indicates $\mathrm{P}<0.01,{ }^{* * *}$ indicates $\mathrm{P}<0.001$. All statistics were calculated using SPSS $®$ Version 25 or GraphPad Prism. Graphs were generated using GraphPad Prism ${ }^{\circledR}$ Version 8.01. For the in vitro experiments, box and whisker plots show median (line) interquartile range (box) and extreme values (whiskers). Error bars represent the standard error of the mean (SEM), refer to the results section for specific tests used per experimental procedure.

\section{Results}

\subsection{Effect of Tofacitinib on Bone Marrow MSC CFU-F Potential}

We evaluated the colony-forming properties of the bone marrow cells after low-density plating using standard assays. Tofacitinib had no effect on bone marrow MSC CFU-F potential, overall colony area, or the average size of individual colonies at any of the concentrations tested (Figure 1A-C).

\subsection{The Effect of Tofacitinib on In Vitro MSC Trilineage Differentiation}

To study if tofacitinib affects the multi-lineage potential of MSCs, we studied its effect in osteogenic, chondrogenic, and adipogenic differentiation assays. Treatment during osteogenic induction of MSCs had no noticeable effect on ALP activity after 14 days of differentiation (Figure 1D), and the accumulation of calcium was similarly unaffected (Figure 1E). We also evaluated the effect of tofacitinib on cartilage differentiation. Again, treatment with the JAK inhibitor did not induce a difference in the accumulation of GAGs, pellet formation, or GAG content in chondrogenic pellet cultures (Figure 1F). This was further confirmed with quantitative measurement of GAG production (Figure 1G).

Tofacitinib treatment dose-dependently increased MSC adipogenesis as measured by oil red staining at day 14 (Figure 2A). The median proportion of area occupied by lipid vacuoles in the control was measured at $5.43 \%$, which increased to $9.32 \%$ and $11.76 \%$ in the $1000 \mathrm{nM}$ and $10000 \mathrm{nM}(\mathrm{n}=6, \mathrm{p}<0.05)$ tofacitinib treatment, respectively (Figure 2B) following paired T-tests. These data suggest that JAK inhibition by tofacitinib in MSCs leads to an increase in adipogenesis. 


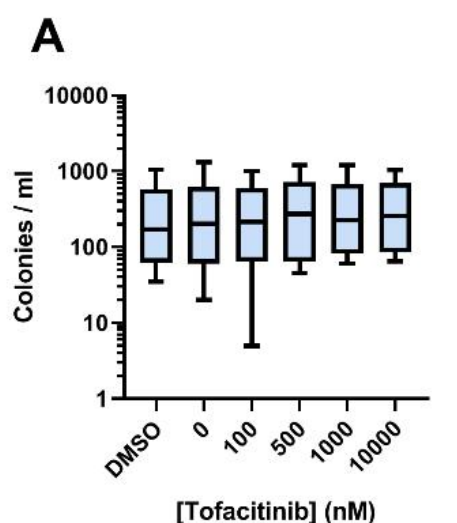

[Tofacitinib] (nM)
B

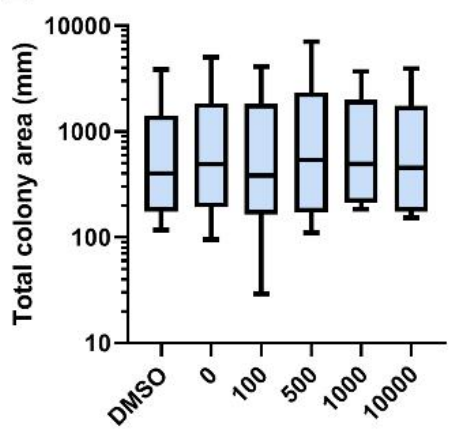

[Tofacitinib] (nM)
C

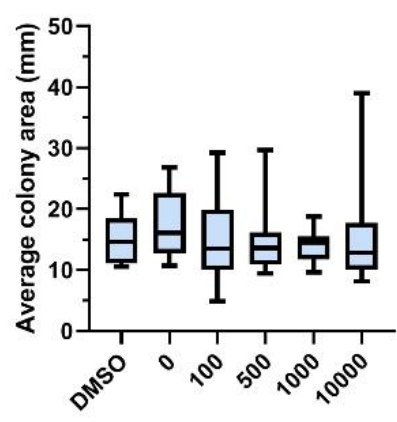

[Tofacitinib] (nM)

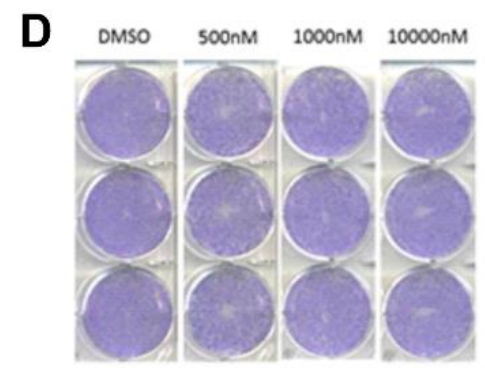

$\mathbf{E}$

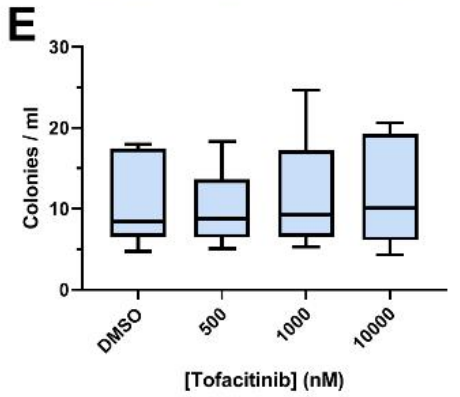

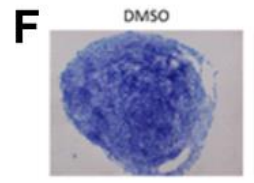

$1000 \mathrm{nM}$

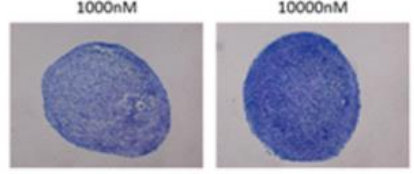

$\mathbf{G}$

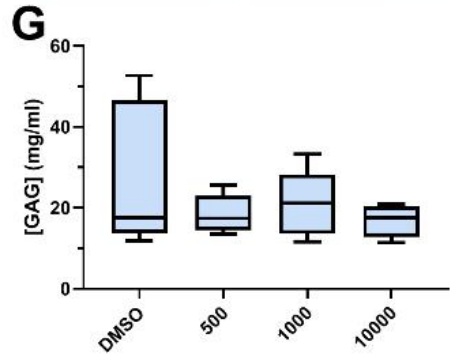

[Tofacitinib] (nM)

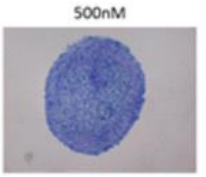

(1)

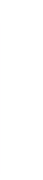

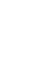

Figure 1. Tofacitinib does not affect MSC colony-forming potential, osteogenesis or chondrogenesis.(A) The effect of tofacitinib on the ability of BM MSCs to initiate adherent colonies and expand in vitro. (B) The effect of tofacitinib on the rate of MSC expansion measured as the total area occupied by colonies, or $(\mathrm{C})$ as the mean area occupied by a single colony. Fast blue staining for ALP activity following osteogenic induction of MSC differentiation in the presence or absence of tofacitinib. Figure shows a single representative donor sample at day 14, in triplicate (D). The effect of tofacitinib on osteogenic differentiation assessed by accumulation of calcium at day 21 (E). The effect of tofacitinib on MSC chondrogenic differentiation, assessed by accumulation of GAGs at day 21. Toluidine blue staining of chondrogenic pellets (F). Quantitative assessment of GAG content in digested chondrogenic pellets $(\mathrm{G})$. 
A

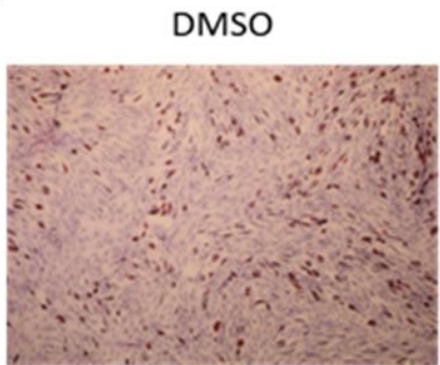

$1000 \mathrm{nM}$

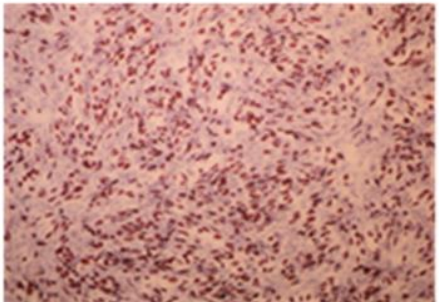

$500 \mathrm{nM}$

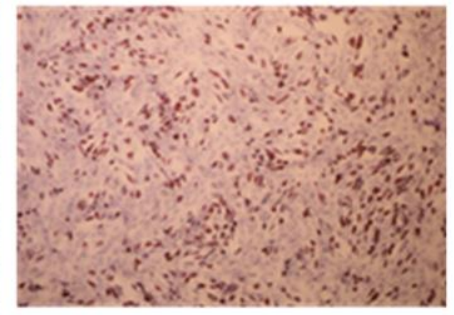

$10000 \mathrm{nM}$

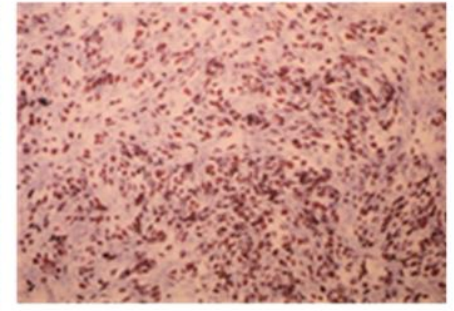

$*$

B

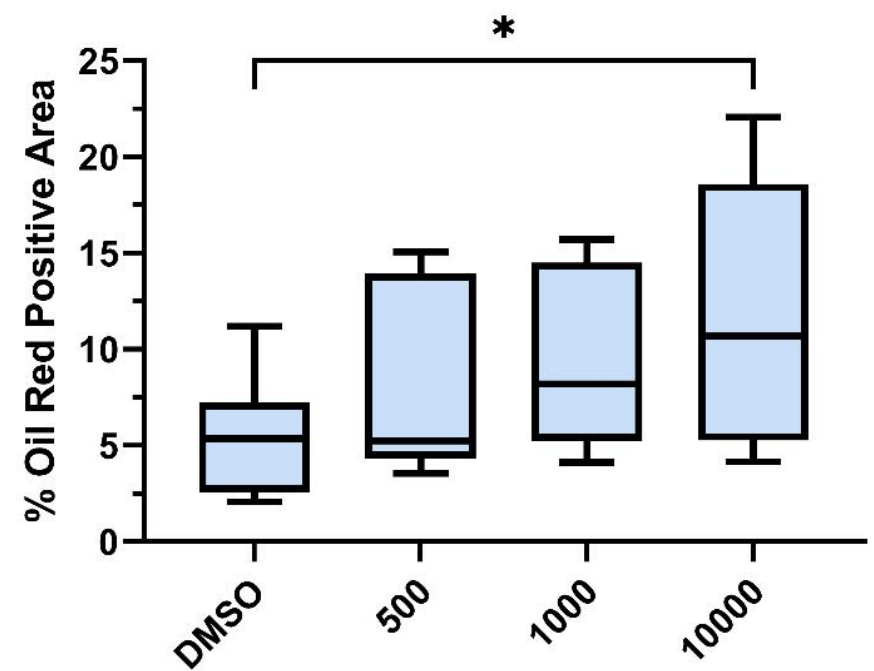

[Tofacitinib] (nM)

Figure 2. Tofacitinib stimulates adipogenesis of MSCs. Representative image showing the effect of tofacitinib treatment on adipogenesis, visualised with the uptake of oil red into lipid vacuoles (red) counterstained with haematoxylin (A). Measurement of oil red stained area of adipogenic cultures at day 14 (B). The median proportion of area occupied by lipid vacuoles in the control was measured at $5.43 \%$, which increased to $11.76 \%$ in the $10000 \mathrm{nM}(\mathrm{n}=6)$ tofacitinib treatment. Paired T-tests. ${ }^{*}=$ $\mathrm{p}<0.05$.

\subsection{Increased Adipogenesis is Linked to Cell Proliferation}

To obtain better insights into the effects of tofacitinib on adipogenesis, we further evaluated the differentiation cultures. Increased fat formation was also shown with Nile red staining (Figure $3 \mathrm{~A})$ with a 2.29 -fold increase $(\mathrm{n}=6, \mathrm{p}<0.05)$ in median fluorescence emission with 10000nM tofacitinib treatment, following Dunn's post-hoc testing (Figure 3B). Using lower concentrations of tofacitinib for 21 days showed a clear dose-dependent increase in fat formation (Figure 3C). A significant increase in Nile red fluorescence was seen for $100 \mathrm{nM}(\mathrm{n}=7, \mathrm{p}<0.05 ; 1.36$-fold $)$ and $500 \mathrm{nM}(\mathrm{n}=7, \mathrm{p}<0.0005 ; 2.10$ fold $)$ tofacitinib treatment relative to the control following Dunn's post-hoc testing. 
We then studied whether tofacitinib affects in vitro pre-adipocyte proliferation and subsequent fat accumulation [33]. Firstly, induction of adipogenesis resulted in a significant increase in DAPI fluorescence reflecting cell numbers compared to control at day 14, for all tofacitinib concentrations (500nM; 1.44-fold, 1000nM; 1.40 and 10000nM; 1.69-fold. $\mathrm{p}=0.028$ ) but with the biggest increase at the lowest $500 \mathrm{nM}$ dose (Figure 4D). A day 21 DAPI signal was significantly increased compared to control in all samples (50nM; 1.07 fold, 100nM; 1.05-fold and 500nM; 1.10-fold $\mathrm{p}<0.05$ for all concentrations) (Figure 3E) except $10 \mathrm{nM}$ treatment. Together with colony-forming assays in which no increases in undifferentiated MSC proliferation were found (Figure 3B), this data suggests that tofacitinib has a positive effect on the proliferation of pre-adipocytes, even in low concentrations.
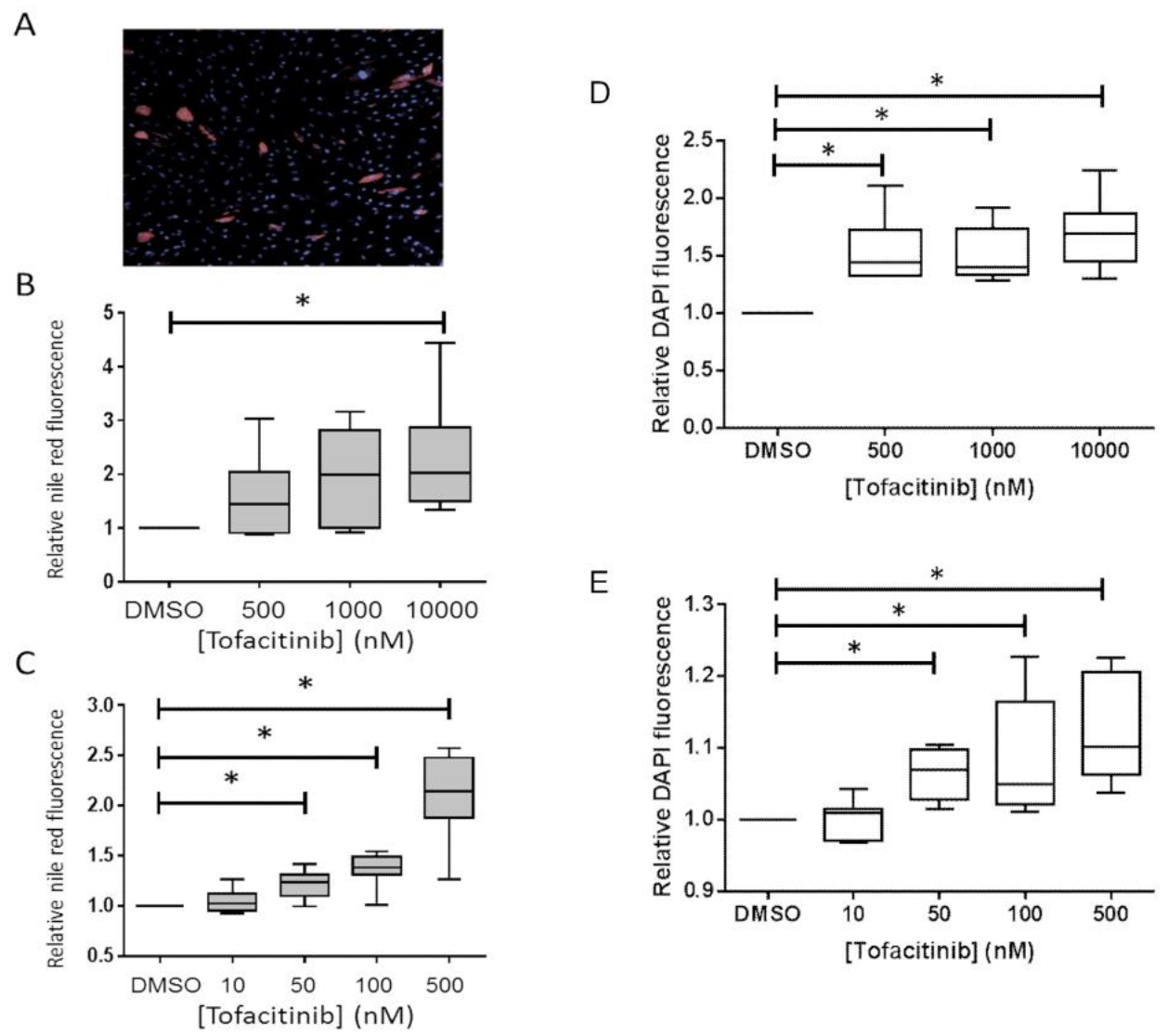

Figure 3. Adipogenic differentiation of MSCs measured by Nile red fluorescence. Representative image showing Nile red uptake into lipid vacuoles (red), counterstained with DAPI (blue) (A). Quantification of Nile red staining at day 14 with high tofacitinib dose (B) $2.29(n=6)$ fold increase in median fluorescence emission with $10000 \mathrm{nM}$ tofacitinib treatment and day 21 with lower tofacitinib treatment dose $(C)$ significance seen in $100(n=7)$ and $500 \mathrm{nM}(n=7)$ tofacitinib treatment relative to the control following Dunn's post-hoc testing. Adipocyte hyperplasia during adipogenic differentiation in the presence of tofacitinib Relative cell number measured by DAPI fluorescence at day 14 (D) (500nM; 1.44-fold, 1000nM; 1.40 and 10000nM; 1.69-fold) and day 21 (E) (50nM; 1.07-fold, 100nM; 1.05 fold and 500nM; 1.10 fold). ${ }^{*}=\mathrm{p}<0.05,{ }^{* * *}=\mathrm{p}<0.001$.

\subsection{Tofacitinib Inhibits Pro-Inflammatory Cytokine Production in An In Vitro Enthesitis Model}

Following the selection of T-cells via magnetic separation, we assessed if tofacitinib [1000nM] had any impact on pro-inflammatory cytokine production, specifically IL-17A and TNF production by ELISA. Following stimulation, tofacitinib [1000nM] effectively inhibited CD4+ and CD8+ T-cell IL-17A and TNF production with the latter showing the most effective inhibition of cytokine production when treated with tofacitinib (Figure 4). 
IL-17A production after CD3/CD28 stimulation of CD4+ T-cells isolated from PBMC was significantly inhibited by tofacitinib treatment $(n=11, p<0.01$, Figure $4 A$ ), this inhibition was also seen in CD4+ T-cells isolated from the PEB $(n=12, p<0.01$, Figure $1 A)$. Though no significant inhibition was seen from the CD8+ T-cell populations at either the PEB or PBMC. CD4+ T-cells from PEB $(n=12, p<0.01)$ and PBMC $(n=11, p<0.01)$ showed significant reductions in TNF secretion after tofacitinib treatment (Figure 4B), with CD8+ T-cells from both PEB $(n=12, p<0.01)$ and PBMC $(n=11, p<0.01)$ showing similar reductions in TNF secretion.

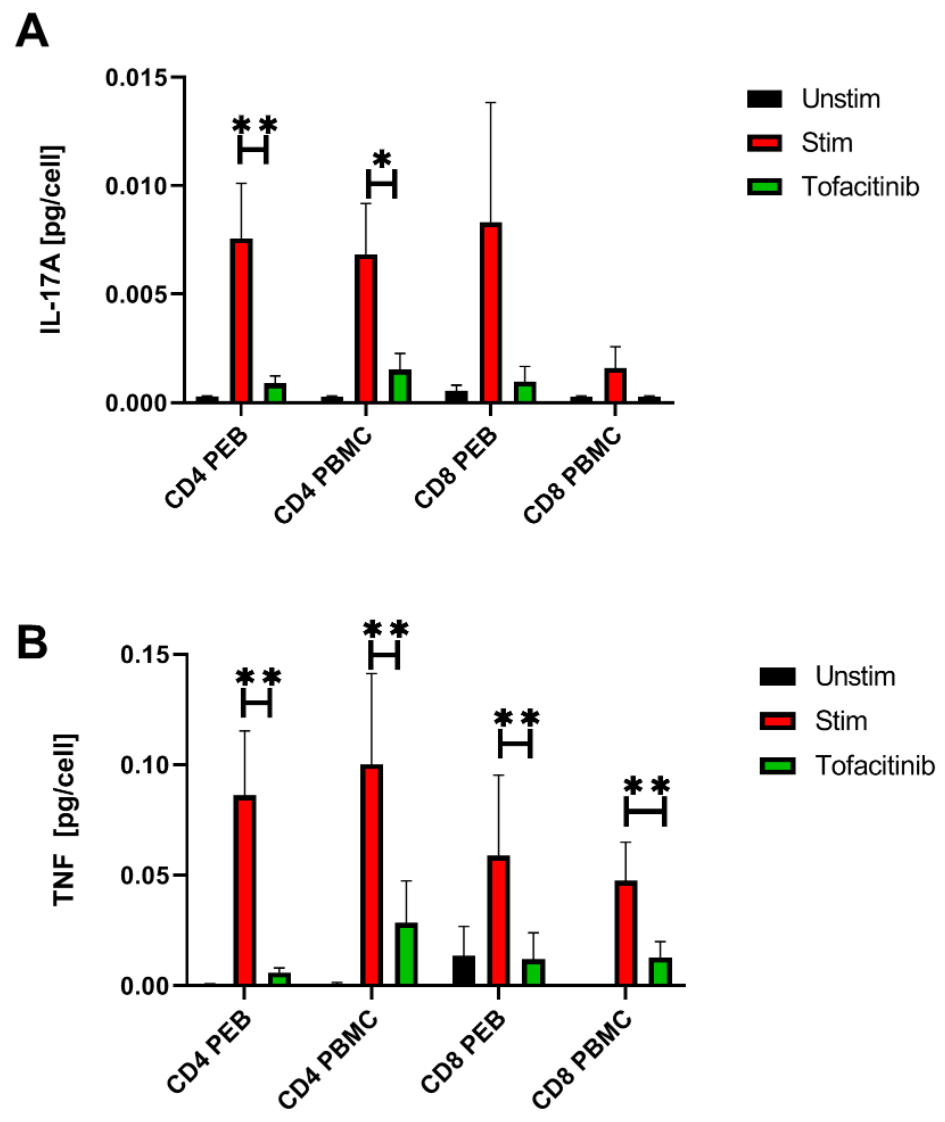

Figure 4. Tofacitinib inhibits pro-inflammatory cytokine production in an in vitro enthesitis model. Following isolation of entheseal CD4+ or CD8+ T-cells, cells were stimulated with anti-CD3/CD28 for $48 \mathrm{hrs}$ with and without 1000nM tofacitinib. IL-17A and TNF secretion were quantified by ELISA with post-hoc Wilcoxon Signed rank testing. CD4+ T-cell from PEB $(n=12)$ and PBMC $(n=11)$ showed significant reductions in IL-17A secretion after tofacitinib treatment when stimulated. All cell populations tested CD4+ T-cells PEB $(n=12)$, PBMC $(n=11)$ and CD8+ T-cells PEB $(n=12)$ and PBMC $(n=11)$ all showed significant reductions in TNF secretion induced by tofacitinib treatment after stimulation. ${ }^{*}=\mathrm{p}<0.05,{ }^{* *}=\mathrm{p}<0.01$.

\section{Discussion}

Adaptive immune cells present at the normal enthesis produce cytokines including TNF and IL-17A that are known to modify MSC function [34]. Accordingly, this work investigated the effect of tofacitinib, a JAK inhibitor, on the differentiation capacity of MSCs and on conventional $\alpha \beta$ CD4+ and CD8+ T-cells in an in vitro enthesitis model and its effect on bone marrow MSCs. Tofacitinib completely blocked IL-17A and TNF protein production from CD4+ and CD8+ T-cells in an in vitro enthesitis model. No effect was shown for MSC differentiation in osteogenic or chondrogenic conditions but tofacitinib did increase MSC adipogenic differentiation. 
In adipogenic conditions, tofacitinib concentrations as low as 50nM were able to significantly increase the lipid content and cellularity of MSCs undergoing in vitro adipogenic differentiation. This is surprising given that studies examining adipogenesis in 3T3L1 cells, a widely used model for adipogenic differentiation [35-37], suggested that STAT5A and STAT5B activation promoted adipogenesis, particularly in the early stages of differentiation, and it could be expected that a JAK inhibitor would antagonise this process [36,38]. Additionally JAK1-STAT3 activation has been implicated in the increased proliferation of pre-adipocytes before terminal differentiation [35,39]. However, pre-adipocytes are responsive to several cytokines that have been shown to inhibit adipogenesis including IFN- $\gamma$ [40], oncostatin M (OSM) [41], and neuropoietin [42], all of which are potent activators of JAK kinases and could suggest an explanation for the results detailed here. However, another cytokine that may explain our results is leptin, which has been identified in bone marrow derived mesenchymal precursors [43] culminating in adipogenesis and inhibition of osteogenic differentiation, interestingly pre-adipocytes from the bone marrow are leptin receptor positive $\left(\mathrm{LepR}^{+}\right)$which signals through JAK2/STAT3 [44].

Further data has shown that adipose-specific disruption of JAK2 and STAT3 signaling in vivo results in increased adipose mass associated with hypertrophy, and increased lipid content $[45,46]$. No such data appear available for JAK1 and JAK3, the preferred targets of tofacitinib. Shi et al found that adipocyte-specific JAK2 deficiency led to impaired lipolysis with aging, suggesting that JAK2 inhibition may cause excessive lipid accumulation in mature adipocytes [46]. This is interesting since hyperlipidemia has been reported as a side effect of tofacitinib treatment in patients with rheumatoid arthritis $[17,18]$. Further investigation is need to elucidate the mechanism by which tofacitinib induces adipogenesis, with respect to signaling pathways.

The ability of tofacitinib to induce adipogenesis in vitro may also have important implications for in vivo osteogenesis and chondrogenesis. The limited in vivo effect may also be understood in this context with JAK1/3 antagonism having an indirect impact on chondro- and osteogenesis. With increased expression of the master transcription factor for adipogenesis PPAR $\gamma$, this suppresses the activation of the master transcription factor for osteogenesis RUNX2 [47]. Lineage of commitment towards adipogenesis requires significantly more DNA histone modification, decreasing osteogenic and chondrogenic differentiations which share an initial differentiation pathway [48].

With regards to the conventional $\alpha \beta$ T-cells, the results support the idea that these entheseal T-cells can secrete pivotal disease-relevant cytokines such as TNF and IL-17A following CD3/CD28 stimulation, without the use of other exogenous cytokines such as IL-23, where in vitro tofacitinib treatment inhibits this production. These results have been reported in other studies showing effective blockade of IL-17A production following tofacitinib therapy [49]. Through murine models and also clinical observations, both TNF and IL-17A have been heavily implicated in enthesitis-related pathology [50-52]. Whilst innate lymphocytes are major inflammatory cytokine producers in mice [53] these findings also suggest that conventional T-cell populations that can express these cytokines are present at the normal enthesis. Given the MHC-I and -II associations with human SpA spectrum disorders, the findings presented here highlight the importance of the investigation of entheseal T-cells in pathological conditions such as AS and PsA. This in vitro system may provide a model for testing the impact of JAK/STAT inhibitors on the adaptive immune system in pre-clinical work in SpA.

IL-17A production has been extensively studied and can either be produced dependently or independently of IL-23 signalling, where the latter involves unconventional innate-like T-cells including MAIT cells (Mucosal associated invariant T), $\gamma \delta$ T cells, type 3 ILC'S (innate lymphoid cells) and iNKT (invariant natural killer T) cells [54-56]. The SKG mouse model which carries a point mutation in the gene encoding the $\mathrm{T}$ cell receptor (TCR)-proximal signaling molecule ZAP-70, develops a T-cell-mediated autoimmune arthritis, which clinically and immunologically resembles SpA in humans [57]. It has been 
reported in previous studies utilising the SKG model that JAK inhibitors including tofacitinib, are effective at reducing pro-inflammatory cytokine production (IL-17A) and increasing immunomodulatory cytokines (IL-10) [58-60]. Where the major arthritogenic Tcell subset within the SKG model, Th17, had its differentiation inhibited by tofacitinib [58]. Interestingly the alternative isoform of the Th17 transcription factor, ROR $\gamma$ has been identified as a negative regulator of adipocyte differentiation when mediated by MMP3 (matrix metalloproteinase 3) in both mice and humans [61]. Due to significant sequence and functional similarities, the ROR subtypes co-expressed in cells may exhibit functional overlap [62].

\section{Conclusions}

In summary, JAK inhibition has a directly inhibited CD4+ and CD8+ T-cells induced IL-17A and TNF production and also MSC relevant transcript inhibition. No impact on MSC osteogenesis was noted but it remains possible that indirect bone effects could occur via the adipogenic pathway. This suggests that clinical trials with JAK/STAT inhibitors in axial SpA should be carefully analyzed for effects concerning adipogenesis as indirectly measured by fatty corner lesions on spinal MRI.

Author Contributions: TR, HR, RC and CB conducted experiments and wrote manuscript. EJ, $\mathrm{DM}, \mathrm{DN}$ contributed to experimental planning, critical discussion of results obtained, as well as manuscript correction.

Acknowledgments: This article/paper/report presents independent research funded/supported by the National Institute for Health Research (NIHR) Leeds Biomedical Research Centre (BRC). The views expressed are those of the author(s) and not necessarily those of the NIHR or the Department of Health and Social Care Funding. The authors have declared no conflicts of interest._RJC is supported by a Pfizer investigator initiated research grant. DM is funded by the Leeds NIHR Biomedical Research Centre, Novartis UK Investigator Initiated non-clinical research funding support (CB, TR, HR). AW was funded by the Celgene supported PARTNER fellowship program.

Institutional Review Board Statement: The study was conducted according to the guidelines of the Declaration of Helsinki and approved by the University of Leeds ethical approval commit-tee. REC: 16/NW/0797.

Informed Consent Statement: Informed consent was obtained from all subjects involved in the study.

Funding: D.M. is funded by the Leeds NIHR Biomedical Research Centre. Funding: Novartis UK Investigator Initiated non-clinical research funding support (H.R., T.R., and C.B.), and research funded by a Pfizer investigator-initiated research grant (R.C.). A.W. was funded by the Celgene supported PARTNER fellowship program.

Conflicts of Interest: The authors declare no conflict of interest.

\section{References}

1. Bridgewood, C.; Watad, A.; Cuthbert, R.J.; McGonagle, D. Spondyloarthritis: New insights into clinical aspects, translational immunology and therapeutics. Current opinion in rheumatology 2018, 30, 526-532.

2. Lories, R.J.; Derese, I.; de Bari, C.; Luyten, F.P. Evidence for uncoupling of inflammation and joint remodeling in a mouse model of spondylarthritis. Arthritis Rheum 2007, 56, 489-497.

3. De Bari, C.; Dell'Accio, F.; Tylzanowski, P.; Luyten, F.P. Multipotent mesenchymal stem cells from adult human synovial membrane. Arthritis Rheum 2001, 44, 1928-1942.

4. De Bari, C.; Dell'Accio, F.; Luyten, F.P. Human periosteum-derived cells maintain phenotypic stability and chondrogenic potential throughout expansion regardless of donor age. Arthritis Rheum 2001, 44, 85-95. 
5. English, A.; Jones, E.A.; Corscadden, D.; Henshaw, K.; Chapman, T.; Emery, P.; McGonagle, D. A comparative assessment of cartilage and joint fat pad as a potential source of cells for autologous therapy development in knee osteoarthritis. Rheumatology (Oxford, England) 2007, 46, 1676-1683.

6. Jones, E.A.; Crawford, A.; English, A.; Henshaw, K.; Mundy, J.; Corscadden, D.; Chapman, T.; Emery, P.; Hatton, P.; McGonagle, D. Synovial fluid mesenchymal stem cells in health and early osteoarthritis: Detection and functional evaluation at the single-cell level. Arthritis Rheum 2008, 58, 1731-1740.

7. Jones, E.; Churchman, S.M.; English, A.; Buch, M.H.; Horner, E.A.; Burgoyne, C.H.; Reece, R.; Kinsey, S.; Emery, P.; McGonagle, D., et al. Mesenchymal stem cells in rheumatoid synovium: Enumeration and functional assessment in relation to synovial inflammation level. Annals of the rheumatic diseases 2010, 69, 450-457.

8. Lories, R.J.; Derese, I.; Luyten, F.P. Modulation of bone morphogenetic protein signaling inhibits the onset and progression of ankylosing enthesitis. J Clin Invest 2005, 115, 1571-1579.

9. Schett, G.; Lories, R.J.; D'Agostino, M.-A.; Elewaut, D.; Kirkham, B.; Soriano, E.R.; McGonagle, D. Enthesitis: From pathophysiology to treatment. Nature Reviews Rheumatology 2017, 13, 731.

10. Pittenger, M.F.; Discher, D.E.; Péault, B.M.; Phinney, D.G.; Hare, J.M.; Caplan, A.I. Mesenchymal stem cell perspective: Cell biology to clinical progress. NPJ Regen Med 2019, 4, 22.

11. Cuthbert, R.J.; Fragkakis, E.M.; Dunsmuir, R.; Li, Z.; Coles, M.; Marzo-Ortega, H.; Giannoudis, P.V.; Jones, E.; El-Sherbiny, Y.M.; McGonagle, D. Brief report: Group 3 innate lymphoid cells in human enthesis. Arthritis Rheumatol 2017, 69, 1816-1822.

12. Cuthbert, R.J.; Watad, A.; Fragkakis, E.M.; Dunsmuir, R.; Loughenbury, P.; Khan, A.; Millner, P.A.; Davison, A.; MarzoOrtega, H.; Newton, D., et al. Evidence that tissue resident human enthesis gammadeltat-cells can produce il-17a independently of il-23r transcript expression. Annals of the rheumatic diseases 2019, 78, 1559-1565.

13. Watad, A.; Rowe, H.; Russell, T.; Zhou, Q.; Anderson, L.K.; Khan, A.; Dunsmuir, R.; Loughenbury, P.; Borse, V.; Rao, A., et al. Normal human enthesis harbours conventional $\mathrm{cd} 4+$ and $\mathrm{cd} 8+\mathrm{t}$ cells with regulatory features and inducible il-17a and tnf expression. Annals of the rheumatic diseases 2020, 79, 1044-1054.

14. Furesi, G.; Fert, I.; Beaufrère, M.; Araujo, L.M.; Glatigny, S.; Baschant, U.; von Bonin, M.; Hofbauer, L.C.; Horwood, N.J.; Breban, M., et al. Rodent models of spondyloarthritis have decreased white and bone marrow adipose tissue depots. Frontiers in Immunology 2021, 12.

15. Veale, D.J.; McGonagle, D.; McInnes, I.B.; Krueger, J.G.; Ritchlin, C.T.; Elewaut, D.; Kanik, K.S.; Hendrikx, T.; Berstein, G.; Hodge, J. The rationale for janus kinase inhibitors for the treatment of spondyloarthritis. Rheumatology 2019, 58, 197-205.

16. Burmester, G.R.; Blanco, R.; Charles-Schoeman, C.; Wollenhaupt, J.; Zerbini, C.; Benda, B.; Gruben, D.; Wallenstein, G.; Krishnaswami, S.; Zwillich, S.H., et al. Tofacitinib (cp-690,550) in combination with methotrexate in patients with active rheumatoid arthritis with an inadequate response to tumour necrosis factor inhibitors: A randomised phase 3 trial. Lancet (London, England) 2013, 381, 451-460.

17. van Vollenhoven, R.F.; Fleischmann, R.; Cohen, S.; Lee, E.B.; Garcia Meijide, J.A.; Wagner, S.; Forejtova, S.; Zwillich, S.H.; Gruben, D.; Koncz, T., et al. Tofacitinib or adalimumab versus placebo in rheumatoid arthritis. The New England journal of medicine 2012, 367, 508-519.

18. Fleischmann, R.; Kremer, J.; Cush, J.; Schulze-Koops, H.; Connell, C.A.; Bradley, J.D.; Gruben, D.; Wallenstein, G.V.; Zwillich, S.H.; Kanik, K.S., et al. Placebo-controlled trial of tofacitinib monotherapy in rheumatoid arthritis. The New England journal of medicine 2012, 367, 495-507.

19. Gao, W.; McGarry, T.; Orr, C.; McCormick, J.; Veale, D.J.; Fearon, U. Tofacitinib regulates synovial inflammation in psoriatic arthritis, inhibiting stat activation and induction of negative feedback inhibitors. Annals of the rheumatic diseases 2016, 75, 311-315.

20. O'Shea, J.J.; Schwartz, D.M.; Villarino, A.V.; Gadina, M.; McInnes, I.B.; Laurence, A. The jak-stat pathway: Impact on human disease and therapeutic intervention. Annu Rev Med 2015, 66, 311-328. 
21. Charles-Schoeman, C.; Burmester, G.; Nash, P.; Zerbini, C.A.; Soma, K.; Kwok, K.; Hendrikx, T.; Bananis, E.; Fleischmann, R. Efficacy and safety of tofacitinib following inadequate response to conventional synthetic or biological disease-modifying antirheumatic drugs. Annals of the rheumatic diseases 2016, 75, 1293-1301.

22. Maeshima, K.; Yamaoka, K.; Kubo, S.; Nakano, K.; Iwata, S.; Saito, K.; Ohishi, M.; Miyahara, H.; Tanaka, S.; Ishii, K. The jak inhibitor tofacitinib regulates synovitis through inhibition of interferon - $\gamma$ and interleukin - 17 production by human cd4+t cells. Arthritis \& Rheumatism 2012, 64, 1790-1798.

23. Yan, J.; Zhang, Z.; Yang, J.; Mitch, W.E.; Wang, Y. Jak3/stat6 stimulates bone marrow-derived fibroblast activation in renal fibrosis. Journal of the American Society of Nephrology : JASN 2015, 26, 3060-3071.

24. Gaber, T.; Brinkman, A.C.K.; Pienczikowski, J.; Diesing, K.; Damerau, A.; Pfeiffenberger, M.; Lang, A.; Ohrndorf, S.; Burmester, G.R.; Buttgereit, F., et al. Impact of janus kinase inhibition with tofacitinib on fundamental processes of bone healing. International journal of molecular sciences 2020, 21.

25. van Beuningen, H.M.; de Vries-van Melle, M.L.; Vitters, E.L.; Schreurs, W.; van den Berg, W.B.; van Osch, G.J.; van der Kraan, P.M. Inhibition of tak1 and/or jak can rescue impaired chondrogenic differentiation of human mesenchymal stem cells in osteoarthritis-like conditions. Tissue Eng Part A 2014, 20, 2243-2252.

26. Baraliakos, X.; Boehm, H.; Bahrami, R.; Samir, A.; Schett, G.; Luber, M.; Ramming, A.; Braun, J. What constitutes the fat signal detected by mri in the spine of patients with ankylosing spondylitis? A prospective study based on biopsies obtained during planned spinal osteotomy to correct hyperkyphosis or spinal stenosis. Annals of the rheumatic diseases 2019, 78, 12201225.

27. Cuthbert, R.; Boxall, S.A.; Tan, H.B.; Giannoudis, P.V.; McGonagle, D.; Jones, E. Single-platform quality control assay to quantify multipotential stromal cells in bone marrow aspirates prior to bulk manufacture or direct therapeutic use. Cytotherapy 2012, 14, 431-440.

28. Bridgewood, C.; Russell, T.; Weedon, H.; Baboolal, T.; Watad, A.; Sharif, K.; Cuthbert, R.; Wittmann, M.; Wechalekar, M.; McGonagle, D. The novel cytokine metrnl/il-41 is elevated in psoriatic arthritis synovium and inducible from both entheseal and synovial fibroblasts. Clin Immunol 2019, 208, 108253.

29. Galotto, M.; Berisso, G.; Delfino, L.; Podesta, M.; Ottaggio, L.; Dallorso, S.; Dufour, C.; Ferrara, G.B.; Abbondandolo, A.; Dini, G., et al. Stromal damage as consequence of high-dose chemo/radiotherapy in bone marrow transplant recipients. Exp Hematol 1999, 27, 1460-1466.

30. Jones, E.; English, A.; Churchman, S.M.; Kouroupis, D.; Boxall, S.A.; Kinsey, S.; Giannoudis, P.G.; Emery, P.; McGonagle, D. Large-scale extraction and characterization of $\mathrm{cd} 271+$ multipotential stromal cells from trabecular bone in health and osteoarthritis: Implications for bone regeneration strategies based on uncultured or minimally cultured multipotential stromal cells. Arthritis Rheum 2010, 62, 1944-1954.

31. Jones, E.A.; Kinsey, S.E.; English, A.; Jones, R.A.; Straszynski, L.; Meredith, D.M.; Markham, A.F.; Jack, A.; Emery, P.; McGonagle, D. Isolation and characterization of bone marrow multipotential mesenchymal progenitor cells. Arthritis Rheum 2002, 46, 3349-3360.

Jones, E.A.; English, A.; Henshaw, K.; Kinsey, S.E.; Markham, A.F.; Emery, P.; McGonagle, D. Enumeration and phenotypic characterization of synovial fluid multipotential mesenchymal progenitor cells in inflammatory and degenerative arthritis. Arthritis Rheum 2004, 50, 817-827.

33. Ghaben, A.L.; Scherer, P.E. Adipogenesis and metabolic health. Nat Rev Mol Cell Biol 2019, 20, $242-258$.

34. Russell, T.; Watad, A.; Bridgewood, C.; Rowe, H.; Khan, A.; Rao, A.; Loughenbury, P.; Millner, P.; Dunsmuir, R.; Cuthbert, R., et al. Il-17a and tnf modulate normal human spinal entheseal bone and soft tissue mesenchymal stem cell osteogenesis, adipogenesis, and stromal function. Cells 2021, 10, 341.

35. Deng, J.; Hua, K.; Lesser, S.S.; Harp, J.B. Activation of signal transducer and activator of transcription-3 during proliferative phases of 3t3-11 adipogenesis. Endocrinology 2000, 141, 2370-2376. 
36. Floyd, Z.E.; Stephens, J.M. Stat5a promotes adipogenesis in nonprecursor cells and associates with the glucocorticoid receptor during adipocyte differentiation. Diabetes 2003, 52, 308-314.

37. Zhang, K.; Guo, W.; Yang, Y.; Wu, J. Jak2/stat3 pathway is involved in the early stage of adipogenesis through regulating c/ebpbeta transcription. J Cell Biochem 2011, 112, 488-497.

38. Nanbu-Wakao, R.; Morikawa, Y.; Matsumura, I.; Masuho, Y.; Muramatsu, M.A.; Senba, E.; Wakao, H. Stimulation of 3t3-11 adipogenesis by signal transducer and activator of transcription 5. Mol Endocrinol 2002, 16, 1565-1576.

39. Lowe, C.E.; O'Rahilly, S.; Rochford, J.J. Adipogenesis at a glance. Journal of cell science 2011, 124, $2681-2686$.

40. Gregoire, F.; De Broux, N.; Hauser, N.; Heremans, H.; Van Damme, J.; Remacle, C. Interferon-gamma and interleukin-1 beta inhibit adipoconversion in cultured rodent preadipocytes. J Cell Physiol 1992, 151, 300-309.

41. Song, H.Y.; Jeon, E.S.; Kim, J.I.; Jung, J.S.; Kim, J.H. Oncostatin m promotes osteogenesis and suppresses adipogenic differentiation of human adipose tissue-derived mesenchymal stem cells. J Cell Biochem 2007, 101, 1238-1251.

42. White, U.A.; Stewart, W.C.; Mynatt, R.L.; Stephens, J.M. Neuropoietin attenuates adipogenesis and induces insulin resistance in adipocytes. The Journal of biological chemistry 2008, 283, 22505-22512.

43. Yue, R.; Zhou, B.O.; Shimada, I.S.; Zhao, Z.; Morrison, S.J. Leptin receptor promotes adipogenesis and reduces osteogenesis by regulating mesenchymal stromal cells in adult bone marrow. Cell Stem Cell 2016, 18, 782-796.

44. Zhou, B.O.; Yue, R.; Murphy, M.M.; Peyer, J.G.; Morrison, S.J. Leptin-receptor-expressing mesenchymal stromal cells represent the main source of bone formed by adult bone marrow. Cell Stem Cell 2014, 15, 154-168.

45. Cernkovich, E.R.; Deng, J.; Bond, M.C.; Combs, T.P.; Harp, J.B. Adipose-specific disruption of signal transducer and activator of transcription 3 increases body weight and adiposity. Endocrinology 2008, 149, 1581-1590.

46. Shi, S.Y.; Luk, C.T.; Brunt, J.J.; Sivasubramaniyam, T.; Lu, S.Y.; Schroer, S.A.; Woo, M. Adipocyte-specific deficiency of janus kinase (jak) 2 in mice impairs lipolysis and increases body weight, and leads to insulin resistance with ageing. Diabetologia 2014, 57, 1016-1026.

47. Stechschulte, L.A.; Lecka-Czernik, B. Reciprocal regulation of ppar $\gamma$ and runx2 activities in marrow mesenchymal stem cells: Fine balance between p38 mapk and protein phosphatase 5. Curr Mol Biol Rep 2017, 3, 107-113.

48. Rauch, A.; Haakonsson, A.K.; Madsen, J.G.S.; Larsen, M.; Forss, I.; Madsen, M.R.; Van Hauwaert, E.L.; Wiwie, C.; Jespersen, N.Z.; Tencerova, M., et al. Osteogenesis depends on commissioning of a network of stem cell transcription factors that act as repressors of adipogenesis. Nature genetics 2019, 51, 716-727.

49. Krueger, J.; Clark, J.D.; Suárez-Fariñas, M.; Fuentes-Duculan, J.; Cueto, I.; Wang, C.Q.; Tan, H.; Wolk, R.; Rottinghaus, S.T.; Whitley, M.Z., et al. Tofacitinib attenuates pathologic immune pathways in patients with psoriasis: A randomized phase 2 study. The Journal of allergy and clinical immunology 2016, 137, 1079-1090.

50. Sherlock, J.P.; Joyce-Shaikh, B.; Turner, S.P.; Chao, C.C.; Sathe, M.; Grein, J.; Gorman, D.M.; Bowman, E.P.; McClanahan, T.K.; Yearley, J.H., et al. Il-23 induces spondyloarthropathy by acting on ror-gammat+ cd3+cd4-cd8-entheseal resident $t$ cells. Nat Med 2012, 18, 1069-1076.

51. Lubrano, E.; Massimo Perrotta, F.; Manara, M.; D'Angelo, S.; Addimanda, O.; Ramonda, R.; Punzi, L.; Olivieri, I.; Salvarani, C.; Marchesoni, A. Predictors of loss of remission and disease flares in patients with axial spondyloarthritis receiving antitumor necrosis factor treatment: A retrospective study. The Journal of rheumatology 2016, 43, 1541-1546.

52. Jacques, P.; Lambrecht, S.; Verheugen, E.; Pauwels, E.; Kollias, G.; Armaka, M.; Verhoye, M.; Van der Linden, A.; Achten, R.; Lories, R.J. Proof of concept: Enthesitis and new bone formation in spondyloarthritis are driven by mechanical strain and stromal cells. Annals of the rheumatic diseases 2014, 73, 437-445.

53. Reinhardt, A.; Yevsa, T.; Worbs, T.; Lienenklaus, S.; Sandrock, I.; Oberdörfer, L.; Korn, T.; Weiss, S.; Förster, R.; Prinz, I. Interleukin-23-dependent $\gamma / \delta \mathrm{t}$ cells produce interleukin-17 and accumulate in the enthesis, aortic valve, and ciliary body in mice. Arthritis \& rheumatology (Hoboken, N.J.) 2016, 68, 2476-2486. 
54. Godfrey, D.I.; Uldrich, A.P.; McCluskey, J.; Rossjohn, J.; Moody, D.B. The burgeoning family of unconventional t cells. Nature immunology 2015, 16, 1114-1123.

55. Cole, S.; Murray, J.; Simpson, C.; Okoye, R.; Tyson, K.; Griffiths, M.; Baeten, D.; Shaw, S.; Maroof, A. Interleukin (il)-12 and il-18 synergize to promote mait cell il-17a and il-17f production independently of il-23 signaling. Frontiers in immunology 2020, 11, 2992.

56. Lee, J.S.; Tato, C.M.; Joyce-Shaikh, B.; Gulen, M.F.; Cayatte, C.; Chen, Y.; Blumenschein, W.M.; Judo, M.; Ayanoglu, G.; McClanahan, T.K. Interleukin-23-independent il-17 production regulates intestinal epithelial permeability. Immunity 2015, $43,727-738$.

57. Sakaguchi, N.; Takahashi, T.; Hata, H.; Nomura, T.; Tagami, T.; Yamazaki, S.; Sakihama, T.; Matsutani, T.; Negishi, I.; Nakatsuru, S., et al. Altered thymic t-cell selection due to a mutation of the zap-70 gene causes autoimmune arthritis in mice. Nature 2003, 426, 454-460.

58. Oh, K.; Seo, M.W.; Kim, I.G.; Hwang, Y.I.; Lee, H.Y.; Lee, D.S. Cp-690550 treatment ameliorates established disease and provides long-term therapeutic effects in an skg arthritis model. Immune Netw 2013, 13, 257-263.

59. Sendo, S.; Saegusa, J.; Yamada, H.; Nishimura, K.; Morinobu, A. Tofacitinib facilitates the expansion of myeloid-derived suppressor cells and ameliorates interstitial lung disease in skg mice. Arthritis research $\mathcal{E}$ therapy 2019, $21,184$.

60. Gracey, E.; Hromadová, D.; Lim, M.; Qaiyum, Z.; Zeng, M.; Yao, Y.; Srinath, A.; Baglaenko, Y.; Yeremenko, N.; Westlin, W. Tyk2 inhibition reduces type 3 immunity and modifies disease progression in murine spondyloarthritis. The Journal of clinical investigation 2020, 130.

61. Meissburger, B.; Ukropec, J.; Roeder, E.; Beaton, N.; Geiger, M.; Teupser, D.; Civan, B.; Langhans, W.; Nawroth, P.P.; Gasperikova, D., et al. Adipogenesis and insulin sensitivity in obesity are regulated by retinoid-related orphan receptor gamma. EMBO molecular medicine 2011, 3, 637-651.

62. Jetten, A.M. Retinoid-related orphan receptors (rors): Critical roles in development, immunity, circadian rhythm, and cellular metabolism. Nucl Recept Signal 2009, 7, e003. 